\title{
Antibacterial Prescription Pattern in Medical and Surgical Intensive Care Units of a Tertiary Care Hospital
}

\author{
Authors \\ *Shahid Saache ${ }^{1}$, Sujeet Divhare ${ }^{2}$, Balasaheb Ghongane ${ }^{3}$, Shaheen Shaikh ${ }^{4}$ \\ ${ }^{1}$ Assistant Professor, Department of Pharmacology, Dr V.M. Govt. Medical College, Solapur \\ ${ }^{2}$ Associate Professor, Department of Pharmacology, Grant Medical College, Mumbai \\ ${ }^{3}$ Professor and Head, Department of Pharmacology, BJ Govt. Medical College, Pune \\ ${ }^{4}$ D.S.T.S.Mandal's College of Pharmacy, Solapur \\ *Corresponding Author \\ Shahid Saache
}

Assistant Professor, Department of Pharmacology, Dr V.M. Govt. Medical College, Solapur

Contact: +919503630866, Email: shahidsaache@gmail.com

\section{ABSTRACT}

Objectives: This study was conducted to assess the prescribing pattern of Antibacterial agents in Medical and Surgical ICU of a tertiary care teaching hospital in Maharashtra and to suggest necessary modifications in prescribing Antibacterial drugs to achieve rational therapeutic practices.

Materials and Methods: In this prospective observational study data of the first 1000 eligible consecutive case records of patients admitted to the Medical and Surgical ICUs during the study period of 1 year were taken. The data was then recorded in the proforma and analysed using Microsoft Excel 2013.

Results: $74.1 \%$ and $25.9 \%$ prescriptions were from Medical and Surgical ICU respectively. Majority of MICU patients were elderly more than 60 years and with involvement of cardiovascular system (41.7\%). Most frequently used Antibacterial drugs in MICU and SICU was Ceftriaxone (48.3\%) and Metronidazole (97.2\%) respectively. Polypharmacy was observed in both. Majority of parenteral Antibacterials were prescribed in MICU (71\%) and SICU (91\%) and more than 95\% Antibacterials were prescribed by brand names in both. $88 \%$ Antibacterials from MICU and $83 \%$ from SICU were from national list of essential medicines.

Conclusion: Most Antibacterial prescriptions were made without bacteriological culture and sensitivity testing evidences in MICU (73.8\%) and SICU (42.9\%).

Antibacterial prescriptions were rational with respect to WHO drug use indicators in most patients. But most of the prescriptions were not supported by culture and sensitivity reports, as it was not done in many patients of ICU. The number of Antibacterials prescribed by generic names was low in the ICU and effort must be made to encourage prescribing by generic names.

Keywords: Antibacterial prescription pattern, Intensive care units, Drug utilization studies, Antibiotics in critical care, Rational drug use. 


\section{INTRODUCTION}

Antimicrobial drugs especially antibacterial drugs play an important role in the management of infections. Patients admitted to the ICUs are generally seriously ill and are suffering from chronic critical illnesses. These patients receive multiple medications especially antibacterial agents because of presence of multiple infections. Furthermore, emergence of resistance to these drugs have been an area of interest recently ${ }^{1}$. Various available literatures documented extensive and indiscriminate use of these drugs in ICU setups ${ }^{2,3}$ hence it is important to conduct drug utilization studies in patients admitted to ICUs. ${ }^{4,5}$.

With this state of affairs, this study was conducted to evaluate the antibacterial prescription pattern in medical and surgical intensive care units of a tertiary care teaching hospital.

\section{MATERIALS AND METHODS}

This cross sectional observational study was conducted at government run tertiary care hospital after getting approval from the Institutional Review Board and Ethics Committee. Consecutive 1000 prescriptions from medical intensive care unit (MICU) which is 20 bedded and surgical intensive care unit (SICU) which is 10 bedded, were collected and put into specially designed case record forms (CRFs). The CRFs were analysed for the most commonly prescribed drugs with special preference given to antibacterial agents. Drug utilization parameters were compared between MICU and SICU and significant difference was calculated. For statistical analysis z test was applied.

Inclusion criteria includes patients with age above twelve years, patients admitted in MICU, patients from SICU, patients of either sex or those patients admitted in one calendar year. Patients from other wards and units than MICU and SICU were excluded from study. Prescriptions of were also assessed for bacteriological investigations for prescribing antibacterial drugs.

\section{RESULTS}

Total 1000 consecutive prescriptions from ICU were assessed. Figure 1 indicates distribution of patients with respect to specialty.

Figure 1: Distribution of patients according to specialty

\section{Percentage of patients admitted in MICU and SICU}

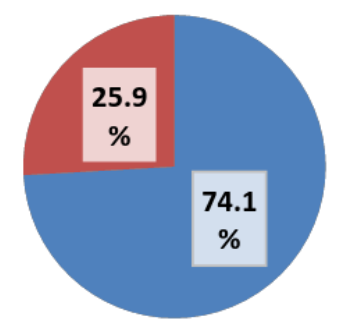

MICU $\quad$ SICU

In MICU $420(56.68 \%)$ were males and 321 $(43.32 \%)$ were females whereas in SICU 139 $(53.67 \%)$ were males and $120(46.33 \%)$ were females. Majority of MICU patients were from age group above 60 years i.e. elderly people.

Age distribution of patients admitted in ICU is given in Table 1.

Table no.1: Age Distribution among patients admitted in MICU and SICU

\begin{tabular}{|c|c|c|c|c|}
\hline$p$ value & $\begin{array}{c}\text { No. of } \\
\text { patients in } \\
\text { SICU } \\
(\mathbf{n}=\mathbf{2 5 9})\end{array}$ & $\begin{array}{c}\text { No. of } \\
\text { patients in } \\
\text { MICU } \\
(n=741)\end{array}$ & $\begin{array}{c}\text { Total } \\
(\mathbf{n}=\mathbf{1 0 0 0})\end{array}$ & $\begin{array}{c}\text { Catego- } \\
\text {-ry } \\
\text { Age }\end{array}$ \\
\hline 0.7444 & $\begin{array}{c}61 \\
(23.55 \%)\end{array}$ & $\begin{array}{c}182 \\
(24.56 \%)\end{array}$ & $\begin{array}{c}243 \\
(24.3 \%)\end{array}$ & $12-30$ years \\
\hline $0.0044 *$ & $\begin{array}{c}81 \\
(31.27 \%)\end{array}$ & $\begin{array}{c}166 \\
(22.40 \%)\end{array}$ & $\begin{array}{c}247 \\
(24.7 \%)\end{array}$ & $31-45$ years \\
\hline 0.9275 & $\begin{array}{c}64 \\
(24.71 \%)\end{array}$ & $\begin{array}{c}181 \\
(24.43 \%)\end{array}$ & $\begin{array}{c}245 \\
(24.5 \%)\end{array}$ & $46-60$ years \\
\hline $0.0106 *$ & $\begin{array}{c}53 \\
(20.46 \%)\end{array}$ & $\begin{array}{c}212 \\
(28.61 \%)\end{array}$ & $\begin{array}{c}265 \\
(26.5 \%)\end{array}$ & $\begin{array}{c}\text { More than } \\
60 \text { years }\end{array}$ \\
\hline
\end{tabular}

$\mathrm{Z}$ test, *p-value $<0.05$ as significant

Prescriptions were further categorized on the basis of predominant systems involved because of which patients got admitted in ICU. System wise distribution of prescription is given in Table 2 
Table no.2: System wise diagnosis

\begin{tabular}{|c|c|c|c|}
\hline $\begin{array}{l}\text { No. of } \\
\text { patients in } \\
\text { SICU }\end{array}$ & $\begin{array}{c}\text { No. of } \\
\text { patients in } \\
\text { MICU }\end{array}$ & $\begin{array}{l}\text { Total no. } \\
\text { of patients } \\
\text { in ICU }\end{array}$ & Systems involved \\
\hline $\begin{array}{l}03 \\
(1.1 \%)\end{array}$ & $\begin{array}{c}309 \\
(41.7 \%)\end{array}$ & $\begin{array}{c}312 \\
(31.2 \%)\end{array}$ & Cardiovascular \\
\hline $\begin{array}{l}18 \\
(6.9 \%) \\
\end{array}$ & $\begin{array}{c}141 \\
(19.0 \%)\end{array}$ & $\begin{array}{c}159 \\
(15.9 \%)\end{array}$ & Respiratory \\
\hline $84(32.4 \%)$ & $\begin{array}{c}124 \\
(16.7 \%)\end{array}$ & $\begin{array}{c}208 \\
(20.8 \%)\end{array}$ & Other \\
\hline $\begin{array}{l}1 \\
(0.3 \%)\end{array}$ & $\begin{array}{c}52 \\
(7 \%)\end{array}$ & $\begin{array}{c}53 \\
(5.3 \%)\end{array}$ & $\begin{array}{c}\text { Central nervous } \\
\text { system }\end{array}$ \\
\hline $\begin{array}{l}0 \\
(0 \%) \\
\end{array}$ & $\begin{array}{c}48 \\
(6.4 \%)\end{array}$ & $\begin{array}{c}48 \\
(4.8 \%)\end{array}$ & Renal \\
\hline $78(30.1 \%)$ & $\begin{array}{c}33 \\
(4.4 \%)\end{array}$ & $\begin{array}{c}111 \\
(11.1 \%)\end{array}$ & $\begin{array}{c}\text { Gastrointestinal/ } \\
\text { Hepatobiliary }\end{array}$ \\
\hline $\begin{array}{l}1 \\
(0.3 \%)\end{array}$ & $\begin{array}{c}22 \\
(3 \%)\end{array}$ & $\begin{array}{c}23 \\
(2.3 \%)\end{array}$ & Hematologic \\
\hline $\begin{array}{l}12 \\
(4.6 \%)\end{array}$ & $\begin{array}{c}12 \\
(1.6 \%)\end{array}$ & $\begin{array}{c}24 \\
(2.4 \%)\end{array}$ & Endocrine \\
\hline $62(23.9 \%)$ & $\begin{array}{c}0 \\
(0 \%)\end{array}$ & $\begin{array}{c}62 \\
(6.2 \%)\end{array}$ & Traumatic \\
\hline $\begin{array}{l}259 \\
(100 \%)\end{array}$ & $741(100 \%)$ & $\begin{array}{c}1000 \\
(100 \%)\end{array}$ & Total \\
\hline
\end{tabular}

Antibacterial drug utilization pattern was studied separately. Antibacterial drugs prescribed and their frequency of their use in MICU and SICU is given in Figure 2 and Figure 3.

Figure 2: Antibacterial drugs prescribed in MICU. Antibacterial drugs prescribed in MICU

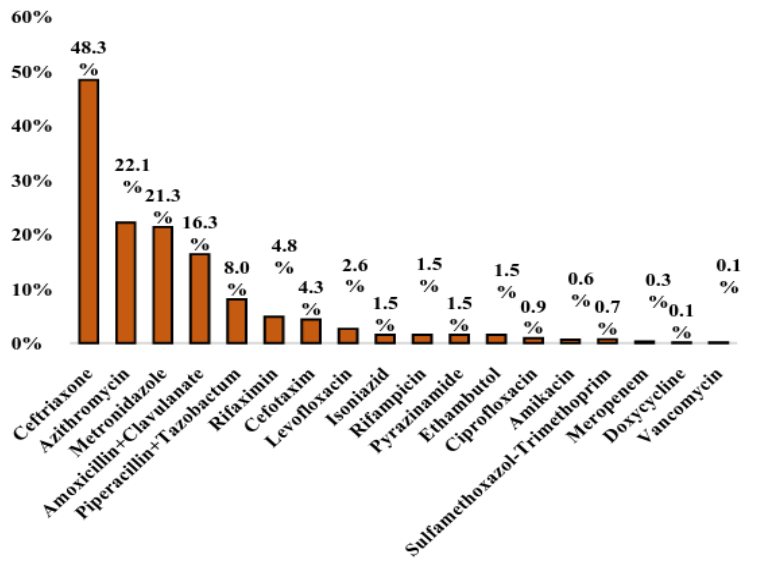

Figure 3: Antibacterial drugs prescribed in SICU Antibacterial drugs prescibed in SICU
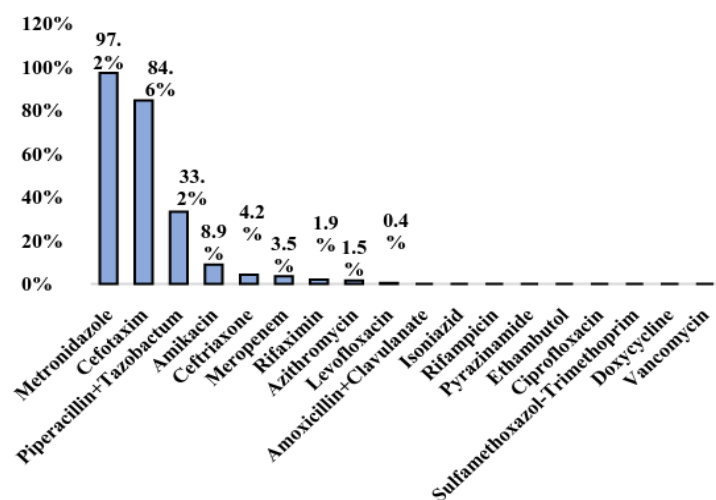

When assessed about number of antibacterial drugs prescribed it was found that most commonly two antibacterial drugs were prescribed in ICU as a total unit i.e.38.3\% of prescriptions and single antibacterial drug was prescribed in $31.4 \%$ of prescriptions. It was found that most of the prescriptions in MICU were consisted of one Antibacterial drug (41.6\%) and most of the prescription from SICU found to include two Antibacterial drugs (61\%).

It was observed that most of the prescriptions from MICU and SICU were prescribed by brand names. Statistically more percentages of prescriptions were found to contain brand names in SICU (98.52\%) than MICU (95.46\%).

Prescriptions were further assessed for their consistency with National list of essential medicines (NLEM). It was also observed that comparatively more number of antibacterial drugs were found to be from NLEM in MICU (88.47\%) than SICU $(83.28 \%)$.

Figure 3 indicates the percentage of patients on antibacterial drugs undergone microbiological investigations to guide the treatment.

Figure 3: Percentage patients on antibacterial drugs and undergone bacteriological culture and sensitivity test.

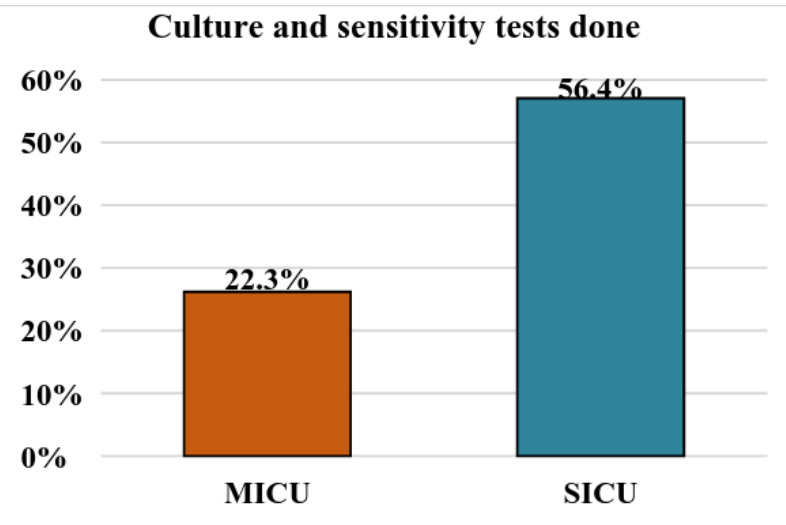

A copy of the National List of Essential Medicines was available at the Department of Medicine and Surgery. 


\section{DISCUSSION}

The study population included patients admitted to MICU and SICU depending on indications. Majority of patients were belong to MICU. Principle reason could be due to number of beds in MICU were double as compared to SICU in this hospital. Furthermore medical conditions requiring admission to ICU are more as compared to surgical indications. In a study conducted by Ahmad Ansari et al ${ }^{6}$ and Mahendra K Patel et al ${ }^{7}$ also found that majority of ICU admission were for medical indications.

In this study more male patients were admitted as compared to female patients in total as well as separately in MICU and SICU. The reason for more male admissions in this study may be attributed to more male to female ratio in Maharashtra and in the Indian scenario. Vandana A Badar et al ${ }^{8}$ in a study got similar results about gender distribution as $57.29 \%$ males and $42.7 \%$ females. Smythe et al ${ }^{46}$ study done outside India did show that equal numbers of male and female patients were admitted to the ICU.

In MICU comparatively more patients were enrolled in the age group of above 60 years i.e. elderly patients. This could be because cardiovascular diseases and other comorbid conditions like diabetes, hypertension etc. are common in such population. Pandiamunian j et al. ${ }^{10}$ and Aparna Williams et al ${ }^{11}$ also found that there were more elderly people in their studies.

Cardiovascular system was most common system involved as a whole as majority of prescriptions belonged to MICU. This could be because of higher prevalence of ischaemic heart diseases in critical care units. Pandiamunian $\mathrm{J}$ et al ${ }^{10}$ also found in their study that ischemic heart disease was most frequent cause of admissions to intensive care department. Nibrad V V et al ${ }^{12}$ also in their study found that most of cases admitted in ICU were related to cardiovascular system and Myocardial infarction and angina pectoris were top clinical diagnoses. In this study prescriptions from SICU were also analysed and found that majority of prescriptions involved Gastrointestinal system and
Hepatobiliary system and many prescriptions were also mentioned head injury as a clinical diagnosis.

On average 1.607 antibacterial drugs were prescribed per prescription. It was found that average number of antibacterial drugs prescribed in MICU and SICU was 1.37 and 2.36 respectively. So usually more than one antibacterial drugs were prescribed to patients admitted in both MICU and SICU. Comparatively more number of antibacterial were used per prescription in SICU than MICU. This reflects patients admitted in SICU were critically ill and suffered multiple infections that require antibiotics to treat. Other studies give figures in ICU not mentioning separately about surgical and medical speciality. The average number of antibiotics per prescription in a similar study in ICU by Mujtaba N Syed et al ${ }^{13}$ and Aparna Williams et al ${ }^{11}$ was 1.60 and 2.09. Most common antibacterial drugs prescribed in MICU were Ceftriaxone followed by Azithromycin. Whereas in SICU most common antibacterial drugs prescribed were Metronidazole followed by Cefotaxime. This could be because rate of anaerobic infection is high in patients admitted to SICU so Metronidazole was given to treat anaerobic infections. Utilization rate of third generation Cephalosporins was very high in both MICU and SICU. This was according to similar studies done in ICUs. Biswal et al 14 reported that the most frequently prescribed antibiotic at ICU admission was metronidazole. Older studies on Antimicrobial prescription pattern found that Penicillins and Aminoglycosides were the most utilized agents. But similar recent studies done in this decade in our country reported that third generation Cephalosporins as the most prescribed Antibacterials in the ICUs 11, 8 . More usage of third generation Cephalosporins may result in Cephalosporin resistant bacteria in this part of the earth ${ }^{15}$.

Polypharmacy with regard to antibacterial was observed in both MICU and SICU. That could be because of serious infections or nosocomial infections ${ }^{9}$.

Most of antibacterial drugs prescribed in both MICU and SICU were in injectable forms. But 
comparatively more percentage of antibacterial drugs were prescribed in injectable form in SICU than MICU. Pandiamunian j et al ${ }^{10}$ also got similar results in their study where $72 \%$ prescriptions found to include injectables.

Most of the antibacterial drugs in both MICU and SICU were prescribed by brand names. Some other studies done in South India also confirmed that the tendency of physicians to prescribe drugs by generic names is less in tertiary care centres and out-patient clinics 16, 17. Prescribing drugs in brand names increase economic burden of the patients as they are costlier than the generic versions available in the market.

About $88 \%$ and $83 \%$ of the prescribed antibacterial drugs were from the National list of Essential Medicines (NLEM) in MICU and SICU respectively. Adhikari et al ${ }^{18}$ and Sreesha et al ${ }^{19}$ have shown that $45.1 \%$ and $56 \%$ of prescribed antimicrobials are consistent with NLEM 2011 which were lower as compared to this study reflecting a better level of adherence to prescribing from NLEM.

About $26 \%$ of patients from MICU and $57 \%$ patients from SICU who were prescribed one or more Antibacterials underwent microbiological investigations. It is recommended to have microbiological tests to know whether organism is sensitive to a particular Antibacterial or not to have proper treatment. It was observed many antibacterials were prescribed without any culture evidence in this study. In contrast to D Chunnilall et al $^{20}$ conducted in corporate ICU, $61.2 \%$ of patients underwent microbiological investigations to guide therapy. So there is a need to have culture based prescriptions of antibacterial drugs.

\section{CONCLUSION}

Majority of Antibacterial drug prescriptions from both MICU and SICU were rational regarding their, route, and the indications but most of them were not supported by culture and sensitivity reports because in this study, culture and sensitivity testing was not done in many patients of both the specialties of ICUs who received Antibacterial drugs. The number of drugs prescribed by generic names was low in both MICU and SICU and effort must be made to encourage prescribing by generic names. Prescribing by generic names will reduce the cost burden on the hospital. The proportion of antibiotic use for bacteriologically proven infections was low. So efforts to prescribe antibiotics on a sound bacteriological basis should be encouraged. So evidence based prescribing should be practice for improved results of critical conditions and infections.

There was an antibiotic policy in tertiary care hospital regarding the use of antibiotics. So proper implementation of antibiotic policy will result in improved clinical outcomes. Proper antibiotic stewardship guidelines should be followed up to tackle the problem of antibiotic drug resistance which is growing very fast due to excessive and inappropriate use of these drugs.

\section{REFERENCES}

1. Samaranayake LP, Johnson NW. Guidelines for the use of antimicrobial agents to minimise development of resistance. Int Dent J. 1999;49(4):189-95.

2. Pandiamunian J, Somasundaram G . A study on prescribing pattern of anti microbial agents in the medical intensive care unit of a tertiary care teaching hospital in puducherry union territory, south india. Int J Pharm Pharm Sci.2014;6(3):235-238.

3. Niederman MS. Appropriate use of Antibacterial agents:Challenges and strategies for improvement. Crit Care Med. 2003;31:608-16.

4. Vandana A Badar, Sanjaykumar B Navale. Study of Prescribing Pattern of Antimicrobial Agents in Medicine Intensive Care Unit of a Teaching Hospital in Central India. JAPI. 2012;60:20-23.

5. Curcio DJ; On behaf of the Latin American antibiotic use in intensive care unit group. Antibiotic prescription in intensive care units in Latin America. Rev Argent Microbiol. 2011;43(3):203-11. 
6. Ahmed Al Ansari et al. Antibiotic Prescription Patterns in an Intensive Care Unit in the Kingdom of Bahrain: An Observational Prospective Study. International $\mathbf{J}$ of scientific research. 2013;2(12):371-74.

7. Mahendra K Patel, Manish J Barvaliya, Tejas K Patel, CB Tripathi. Drug utilization pattern in critical care unit in a tertiary care teaching hospital in India. International $\mathbf{J}$ of Critical Illness and Injury Science. 2011;3(4):250-255.

8. Vandana A Badar, Sanjaykumar B Navale. Study of Prescribing Pattern of Antimicrobial Agents in Medicine Intensive Care Unit of a Teaching Hospital in Central India. JAPI. 2012;60:20-23.

9. Smythe MA, Melendy S, Jahns B, Dmuchowski. An exploratory analysis of medication utilization in a medical intensive care unit. Crit Care Med.1993;21(9):131923.

10. Pandiamunian J, Somasundaram G . A study on prescribing pattern of anti microbial agents in the medical intensive care unit of a tertiary care teaching hospital in puducherry union territory, south india. Int J Pharm Pharm Sci.2014;6(3):235-238.

11. Williams A, Mathai AS, Phillips AS. Antibiotic prescription patterns at admission into a tertiary level intensive care unit in Northern India. J Pharm Bioallied Sci. 2011;3(4):531-6.

12. Nibrad V V, Nayak BB, Raul AR, Vijayprasad S,Vakade KP,Jadhav AR, Vijaykumar AN. Drug utilization pattern in medical intensive care unit (micu) in a tertiary care teaching hospital in rural area of Maharashtra.2015;6(1):148-153.

13. Mujtaba Hussain Naqvi Syed, Chiranjeevi Uday Kumar, and J.C.Shobha. Prescription Patterns of Antibiotics in Acute Medical Care Unit of a Tertiary Care Hospital in India. Int.J.Curr.Microbiol.App.Sci. 2014;3(7):673-679
14. Biswal S, Mishra P, Malhotra S, Puri GD, Pandhi P. Drug utilization pattern in the intensive care unit of a tertiary care hospital. J Clin Pharmacol. 2006;46(8):945-51.

15. Dancer SJ. Problem with cephalosporins. J Antimicrob Chemother. 2001;48(4):463-78.

16. Pandiamunian J, Somasundaram G, Manimekalai K, Kartik JS. A study on prescribing pattern of drugs by general practitioners in a rural area of Tamilnadu. Int J Pharm Bio Sci 2013;4(2):480-6.

17. Pandiamunian J, Ishwarya $\mathrm{T}$, Thivya $\mathrm{T}$, Keerthana A. A questionnaire based study on perception of doctors working in a tertiary care teaching hospital towards their prolonged stay duties in hospitals, ADR reporting tendency, generic drug preferences and their lifestyle. Int $\mathbf{J}$ Pharm Bio Sci 2014;5(1):640-5.

18. Adhikari A, Singh S, Bhowal T, Biswas S, Banerjee S, Ray $M$, et al. Study of prescribing pattern of antimicrobial agents in selected patients attending tertiary care hospital in India. Explor Anim Med Res 2013;3:29-35.

19. Sireesha, Gudapati Bhanu Naga et al. "Antimicrobial Utilization in Multidisciplinary Intensive Care Units of a Public Tertiary Care Hospital." Journal of Pharmacology \& Pharmacotherapeutics. 2014;5(4):252-254.

20. D Chunnilall, AK Peer, I Naidoo, SY Essack. An evaluation of antibiotic prescribing pattern in adult intensive care units in a private hospital in KwaZulu-Natal. South Afr J Infect Dis. 2015;30(1):17-22. 